\title{
GPPS-BJ-2019-0097
}

\section{MOMENTUM TRANSPORTATION ANALYSIS IN AXIAL-SLOT CASING TREATMENT FOR AN AXIAL COMPRESSOR}

\author{
Junda Feng \\ School of Aeronautics and Astronautics, \\ Shanghai Jiao Tong University \\ dasamda@sjtu.edu.cn \\ Shanghai, China \\ Jinfang Teng \\ School of Aeronautics and Astronautics, \\ Shanghai Jiao Tong University \\ tjf@sjtu.edu.cn \\ Shanghai, China
}

\author{
Mingmin Zhu \\ School of Aeronautics and Astronautics, \\ Shanghai Jiao Tong University \\ jackzhushen@sjtu.edu.cn \\ Shanghai, China \\ Xiaoqing Qiang \\ School of Aeronautics and Astronautics, \\ Shanghai Jiao Tong University \\ qiangxiaoqing@sjtu.edu.cn \\ Shanghai, China
}

\begin{abstract}
In this paper, momentum transport analysis was proposed to investigate the effects of axial-slot casing treatment. The numerical simulation was performed on a transonic single rotor with and without casing treatment. The distribution of momentum in the tip region and slot opening was obtained. The result shows that casing treatments suppress the tip leakage flow and improve the axial momentum distribution near the tip. The decomposed momentum distribution demonstrates the flow interaction between casing treatment and tip region. The flow pumped into casing treatment is always with reverse axial momentum and the flow injected from casing treatment is always in positive axial momentum for these semi-circular slots. Furthermore, the influence of casing treatment axial position is also investigated by the analysis of momentum transportation. The numerical simulations were conducted in another two configurations with different axial positions. The results indicate that the axial momentum is not the only parameter that influences the stall margin. The strength of interaction between casing treatment and tip region should be neither too violent nor too weak. There might be an optimal momentum distribution that the demarcation point is just at the leading edge in axial momentum distribution.
\end{abstract}

\section{INTRODUCTION}

The rotating stability is an important issue in the axial compressor. The rotating stall can lead to great damage in turbomachinery. In many cases, the instability is caused by the tip leakage flow. According to (Vo et al., 2008), the spillage flow at the leading edge and the backflow at the trailing edge are two conditions necessary for the formation of the spike- type stall inception, which may trigger a stall in the compressor.

Many methods have been used to control the rotating instability. Casing treatment is found to be an effective passive control method for the instability occurring in the rotor tip since the 1950s. The researchers tested a mass of geometries on different test rigs in the past decades (Bailey and Voit, 1970; Koch, 1970; Brandstetter et al., 2015). The experimental results showed that the casing treatment extended the stall margin but at the cost of efficiency loss. Nowadays, more and more studies are performed by computational fluid dynamics (CFD). Some mechanisms of casing treatment have been proposed (Emmrich et al., 2007; Lu et al., 2009; Muller et al., 2008). After several decades of research, casing treatment has been used in various compressors. However, there is still no general design method for casing treatment. A proper method that can evaluate the effectiveness of casing treatment will help to establish a general design method of casing treatment.

There are two types of casing treatments, the circumferential grooves and the axial slots. The axial slots have a better effect on extending stall margin and are used in more compressors (Bailey and Voit, 1970; Koch, 1970; Moore et al., 1971). As shown by Danner's experiment (Danner et al., 2009), the semi-circular slots have a stall margin extension with the increase of peak efficiency at design condition, which is different from the opinions before. However, such phenomenon was not found in Wilke's experiment (Wilke, 2004). Hence, whether the semi-circular slots have such effects is still unclear, as well as the mechanism of this phenomenon. Further research should be done to explain the phenomenon. 
Momentum analysis has been used successfully in analyzing flow fields of axial compressor. (Du et al., 2010) defined momentum ratio of tip leakage flow to incoming flow in axial direction to compare the relative strength of tip leakage flow and incoming flow. A limit curve of momentum ratio has been proposed to predict the unsteady tip leakage flow, which could lead to compressor stall. (Nan, 2014) investigated the influence of circumferential grooves in Rotor 67 by analyzing the momentum of a specific control volume inside the grooves. Besides, the momentum distribution in the tip clearance is used as a criterion to evaluate the tip leakage flow strength (Cameron et al., 2013; Hewkin-Smith et al., 2019; Nan, 2014). For the circumferential grooves, the momentum flux makes the extension of stall margin linearly (Shabbir and Adamczyk, 2005; Ross et al., 2017). However, the analysis of momentum transportation has not been used to investigate the interaction between tip region and axial-slot type casing treatment.

In this paper, the author proposes momentum transportation analysis method to investigate the interaction between axial slot casing treatment and tip region. In order to have a detailed scene of the flow fields, the momentum is decomposed into axial, tangential and radial directions. By analyzing the profiles of momentum distribution, the flow is described in quantity. Finally, the relationship between stall margin extension and momentum distribution has been established.

\section{TEST ROTOR AND CASING TREATMENT}

The investigated compressor in this research is the transonic compressor stage at Technische Universität Darmstadt, consisting of 16 rotor blades and 29 stator vanes, known as Darmstadt Transonic Compressor (DTC). To exclude the unsteady effect of downstream stators and save computation resources, the stators are omitted in simulations in this paper. This test rotor has 16 blades with aspect ratio of 0.94. The rotating speed is $20000 \mathrm{rpm}$ and the tip Mach number at the inlet is 1.35 . At design work condition, the total pressure ratio is 1.5 with mass flow of $16 \mathrm{~kg} / \mathrm{s}$. Other details of the compressor stage are shown in Table 1 ((Danner et al., 2009)).

Table 1 Design parameters of the DTC compressor stage

\begin{tabular}{lr}
\hline Parameters & Value \\
\hline Mass flow & $16.0 \mathrm{~kg} / \mathrm{s}$ \\
Total pressure ratio & 1.5 \\
Rotating speed & $20000 \mathrm{rpm}$ \\
Tip diameter & $380 \mathrm{~mm}$ \\
Hub-to tip ratio & 0.51 \\
Tip clearance (of chord) & $1.7 \%$ \\
Aspect ratio & 0.94 \\
\hline
\end{tabular}

The semi-circular slots casing treatment (SE) reported in Danner's work (Danner et al., 2009) was employed in the study. 64 axial slots distributed equidistantly around the circumferential direction with 45 degrees leaning towards rotation direction. The axial length of casing treatment (CT) was $42 \mathrm{~mm}$, which is the same as the axial length of rotor tip. Since the slots were semi-circular, the maximum depth of the slots is half of the length, i.e., $21 \mathrm{~mm}$. The porosity in the circumference is $50 \%$, which means the slot width is equal to the distance between two adjacent slots.

Three CT configurations were investigated in the study. Each configuration has the same geometry and porosity. The axial position was the only difference, which leads to different overlap of CT and rotor tip. For the original configuration, the overlap between CT and rotor blade is $33 \%$. The medium overlap $\left(\mathrm{SE}_{\mathrm{M}}\right)$ is described as a $50 \%$ axial overlap between $\mathrm{CT}$ and blade. The third condition is the large overlap $\left(\mathrm{SE}_{\mathrm{L}}\right)$, with an overlap of $66.7 \%$. The diagrams of three configurations are shown in Figure 1 (Danner et al., 2009). According to Danner's experimental results, all three configurations can extend the stall margin effectively. To compare the stall margin conveniently, the stall margin is calculated as

$$
\text { S.M. }=\left(\frac{\pi_{s} / m_{s}}{\pi_{d} / m_{d}}-1\right) \times 100 \%
$$

The configuration SE contributes the best stall margin extension of $40.17 \%$, while $\mathrm{SE}_{\mathrm{M}}$ and $\mathrm{SE}_{\mathrm{L}}$ achieve $25.14 \%$ and $26.42 \%$ of extension.

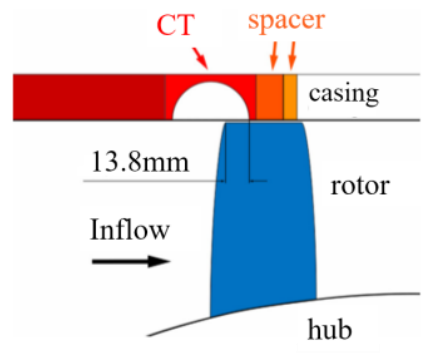

(a) SE

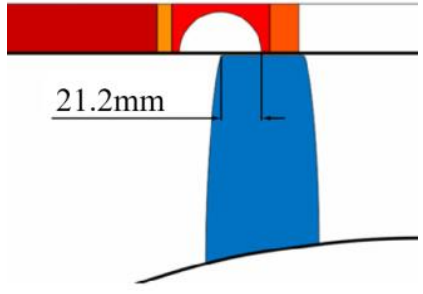

(b) $\mathrm{SE}_{\mathrm{M}}$

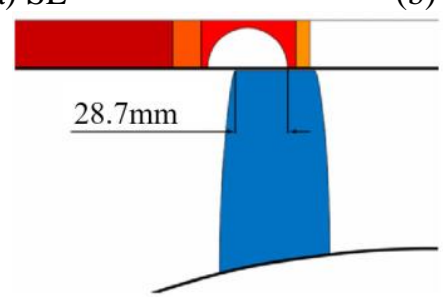

(c) $\mathrm{SE}_{\mathrm{L}}$

Figure 1 Diagrams of SE, SEM, and SEL (Danner et al., 2009)

\section{NUMERICAL SIMULATION}

The ANSYS $®$ CFX 16.0 was used to solve the threedimensional, unsteady Reynolds-Averaged Navier-Stokes equations. The SST turbulence model was chosen in the simulation. Second order backward Euler scheme was used as the temporal discretization for the unsteady simulation, and second order upwind Euler scheme was used as the spatial discretization.

The structural mesh was multiblock and generated by the IGG/AUTOGRID 5 module of NUMECA software, including the blade passage, the casing treatment and a thin slice (3\% of tip clearance) between them as the basis for the rotor/slots 
interface. Figure 2 gives a detailed view of the mesh. The $\mathrm{O} 4 \mathrm{H}$ topology was used to improve the grid quality around the blade. An H-type mesh was used in the thin slice. A butterflytype topology mesh was used in the CT block to refine the orthogonality. It is important to validate the mesh independence, so each block with different number of nodes was checked. For the rotor block, mesh nodes of 182088 , 561668 and 1438728 were tested. After comparison with the experimental results, mesh with 561668 nodes was chosen as the blade passage mesh. In this mesh, the nodes number in radial direction is 73 and in tip leakage is 17 . There are 148 nodes around the blade in the $\mathrm{O}$ type topology. For the CT block and slice block, the detailed comparison was listed in Table 2. Finally, the mesh M2 reached the independence with less nodes number.

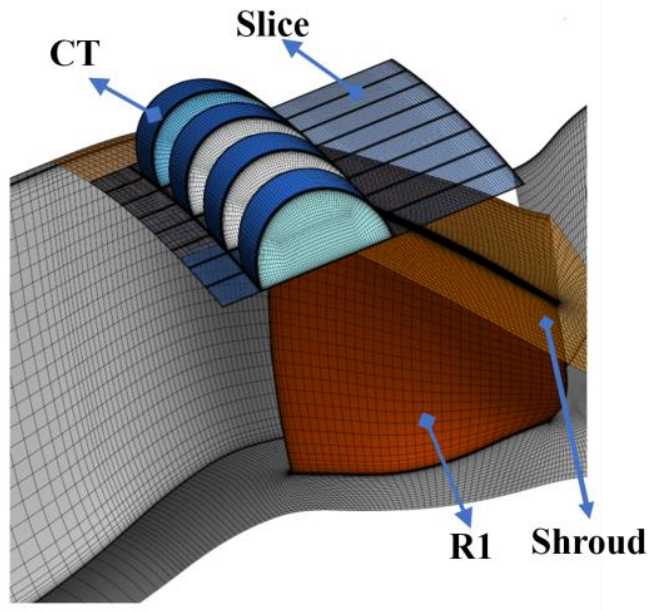

Figure 2 Diagram of computation mesh

Table 2 Details of CT and Slice mesh

\begin{tabular}{lrrr}
\hline & Number of CT & Number of Slice & Total number \\
\hline M1 & 11492 & 47045 & 93013 \\
M2 & 44100 & 221697 & 398097 \\
M3 & 111012 & 611325 & 1055373 \\
\hline
\end{tabular}

Since the effect of casing treatment is unsteady, the steady simulation cannot reflect the interaction of casing treatment flow and main flow. Hence, the unsteady simulation was also conducted. To obtain an accurate solution, the validation of time step independence was conducted. Two time steps which are $1 / 48$ and $1 / 96$ blade passing period were compared. The result shows that the performance changing with time is almost the same for both time steps. Finally, the time step is chosen as $1 / 48$ blade passing period $\left(3.90625 \times 10^{-6} \mathrm{~s}\right)$ in the simulation. More than 5000 time-steps were performed that sufficient rotor period was simulated.

At the inlet of the rotor, total pressure and total temperature of the standard air were imposed as the boundary condition with flow direction normal to inlet plane. The outlet boundary condition was corrected mass flow rate. The corrected mass flow rate was gradually reduced to reach the stall condition. No-slip and adiabatic condition were imposed as the solid wall boundary. The interface between CT and blade passage was transient rotor-stator, which can capture the unsteady flow feature with a high cost of time and computation resource.

\section{RESULTS AND DISCUSSION}

\subsection{Compressor performance}

The compressor performance maps of both smooth casing (SC) and SE configurations are shown in Figure 3, which are achieved by steady and unsteady simulations. The experimental results obtained in the stage environment (Danner et al., 2009) are also proposed. EXP is the abbreviation of experiment and SIM is simulation. In SC configuration, the near stall point in experiment is about 14 $\mathrm{kg} / \mathrm{s}$, and the near stall point in simulation is about $14.67 \mathrm{~kg} / \mathrm{s}$. In SE configuration, the near stall point in experiment is around $12.5 \mathrm{~kg} / \mathrm{s}$, and the near stall point in simulation is about $13.24 \mathrm{~kg} / \mathrm{s}$.

The results of the numerical simulation and experiment are well matched in most work point for both SC and SE, though there is a difference in the near stall point. Therefore, the simulation results can be regarded as reliable. Besides, the near stall point is at a low mass flow rate for the rotor with casing treatment, i.e., the casing treatments extend the stall margin. Notice that the stall point of unsteady simulation is different from the steady result. The unsteady feature of casing treatment is responsible for such a difference. Moreover, the stall point of unsteady simulation has a larger mass flow rate than the experiment result. The single passage model is one of the reasons for the difference. The stall cell propagates in tangential direction. But the boundary condition in the tangential direction is periodical in the single passage model, which is different from experiment condition. Though the single passage simulation has its limitation, the calculated tip flow fields are reasonable. Thus it is practicable to perform a momentum transportation analysis with single passage simulation.

The work condition for analysis in the following part is at the $14 \mathrm{~kg} / \mathrm{s}$ mass flow rate, which is the near stall point of SC configuration.

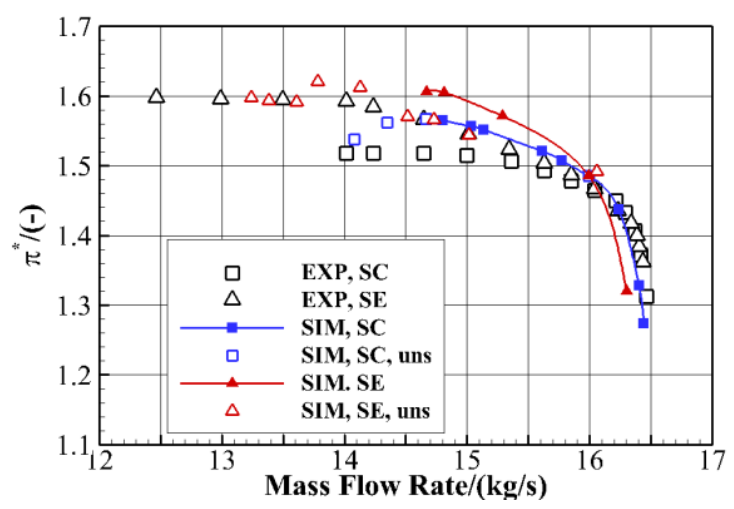

Figure 3 Compressor performance of SC and SE 


\subsection{Momentum analysis at rotor tip between SC and SE cases}

The analysis of momentum transportation is a powerful tool in analyzing the flow character in the tip region. The method takes both mass flow and velocity into consideration, which is more reasonable than only velocity. The axial momentum distribution $\alpha$ can be defined as

$$
\alpha=\frac{\left|\rho_{L} v_{L, \text { axial }}\right| v_{L, \text { radial }}}{\dot{m}_{\text {in }} v_{x, \text { in }} / c_{x, \text { tip }} / \tau}
$$

where the subscript $L$ means the leakage flow and $\tau$ is the pitch distance between two rotor tips. Therefore, $\alpha$ is the normalized momentum distribution per unit area. The flow parameters used in the equation are time-averaged during a short time. Figure 4 shows the momentum distribution on the tip surface at $99 \%$ span, without casing treatment (a) and with casing treatment (b) at the same mass flow condition.

In Figure 4 the trajectory of tip leakage vortex is revealed clearly. A figure of $C_{P}$ is also illustrated as Figure 5 to show the trajectory of tip leakage vortex. $C_{P}$ is calculated as

$$
C_{P}=\frac{P-P_{i n}}{P_{i n}^{*}-P_{i n}}
$$

Compared Figure 4 with Figure 5, the trajectory shown by two different way fits well. As Figure 4 shows, the momentum of leakage flow is below zero, which means leakage flow has a direction against the main flow. Moreover, the largest value of the reverse flow momentum is around 0.5 , which is near an order of magnitude larger than the main flow. The vortex trajectory propagates from leading edge to more than a half of the blade passage in tangential direction and $24.39 \%$ of tip chord in axial direction. As a result, the reverse leakage flow establishes blocking region, which is an inducement of the rotating stall. Figure 4(b) represents the distribution of axial momentum with SE. The influence of casing treatment SE improves the flow field in the tip region. Upstream the leading edge, there are several regions with high value of momentum along the positive direction. Fluid in this region is injected by the casing treatment, which forces the leakage flow to move downstream. Compared with SC configuration, the leakage flow is suppressed by the injected fluid. The end of the vortex trajectory moves downstream near $9 \%$ of the tip chord. The circumferential influence of leakage flow was also suppressed. Moreover, the largest value of the reverse flow momentum decreases less than 0.45 , and the largest value of main flow increases to near 0.198 .

With the help of momentum analysis, the flow feature between the tip leakage flow and the main flow is manifested clearly.

\subsection{Momentum distribution on the slot opening}

In the previous section, the momentum distribution on the tip region shows that the tip leakage flow plays an important role in the rotation stall. In this section, a similar analysis is used for the slot opening, to investigate the casing treatments from a different perspective. Different from the last section, the momentum distribution in this section is along the axial direction instead of a 2-D distribution. The momentum decomposed in specific direction is defined as

$$
\mu_{C T, i}=\int_{L_{0}}^{L_{1}} \frac{\left|\rho_{C T} v_{C T, r}\right| v_{C T, i}}{m_{i n} v_{x, i n} / c_{x, t i p}} d L
$$

where the subscript $C T$ is the abbreviation of casing treatment, $i$ is the specific direction, which can be axial (subscript $\mathrm{x}$ ), tangential (subscript t) and radial (subscript r) directions. $L$ is the circumferential width of the slot, and $L_{0}$ is the start of the integration while $L_{1}$ is the end (the detail of integration location is shown in Figure 6).

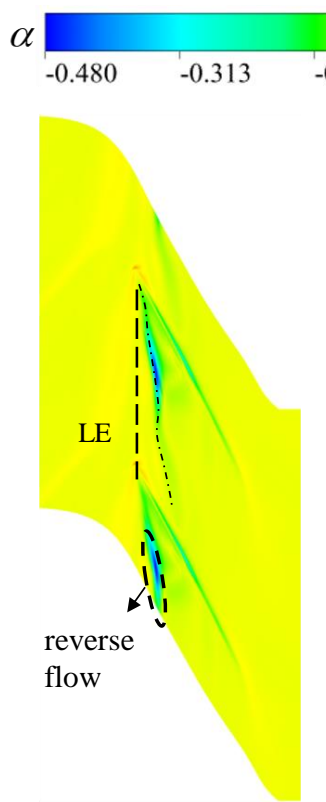

(a) $\mathrm{SC}$

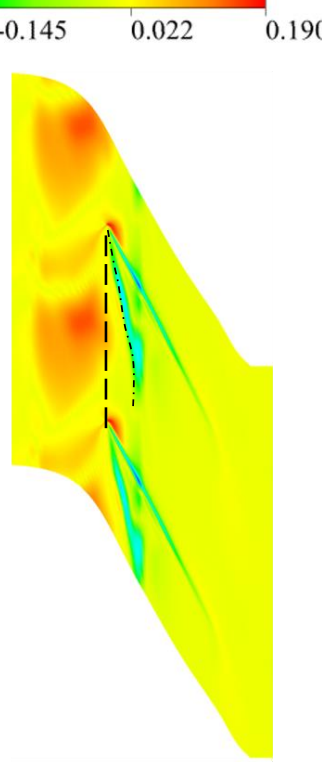

(b) SE
Figure 4 Axial momentum distribution on $99 \%$ span

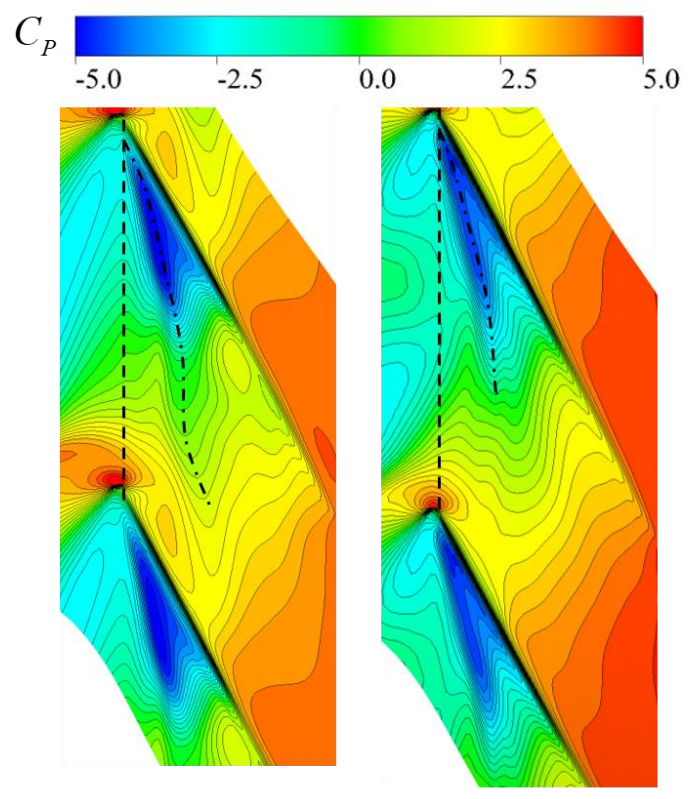

(a) $\mathrm{SC}$

(b) $\mathrm{SE}$

Figure 5 Tip leakage trajectory revealed by $C_{P}$ 


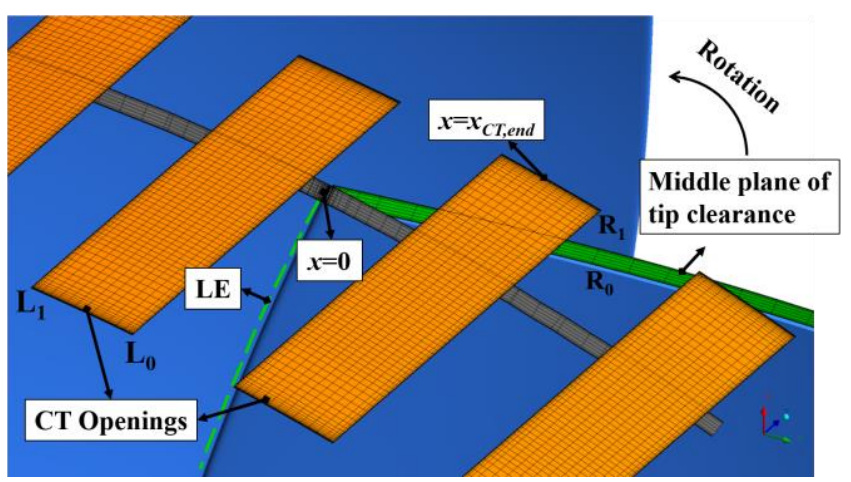

Figure 6 The integration plane

Figure 7 shows the time-averaged distribution of mass flow rate, velocity and the momentum in three directions. Mass flow normalized by the inlet mass flow rate is shown in Figure 7(a). The mass flow rate larger than 0 represents injection into the slots. The leading edge of rotor tip is critical. Upstream the leading edge, flow moves from casing treatment to blade passage. On the contrary, downstream the leading edge, the flow mainly sucks from the tip region. At $25 \% c_{x}$, tip downstream the leading edge, the flow into slots reaches the maximum equal to $2 \%$ inlet mass flow. Correspondingly, the flow injected to the blade passage reaches maximum at $-40 \%$ $c_{x, \text { tip }}$ with $1 \%$ inlet mass flow.

The normalized velocity distribution is also illustrated, which is normalized by the tangential velocity at tip region. The radial velocity with positive value signifies flow moves into the slots. The positive direction of tangential velocity is the same as the rotating direction. Similar to the mass flow rate, the leading edge is still a critical point of velocity profile. Upstream the leading edge, $\bar{v}_{C T, x}$, and $\bar{v}_{C T, t}$ are positive value, while $\bar{v}_{C T, r}$ is negative value. The velocity profile downstream the leading edge is just opposite to the upstream.

The profiles of momentum in different directions are analogous to the velocity, with a similar trend along the axial direction. All the velocity components reach the maximum near $25 \% c_{x, \text { tip }}$ mainly because the mass flow rate reaches the largest value there. Besides, the momentum is near zero between $-20 \%-5 \% c_{x, t i p}$. Reason for this is that the mass flow rate near the range is around zero.

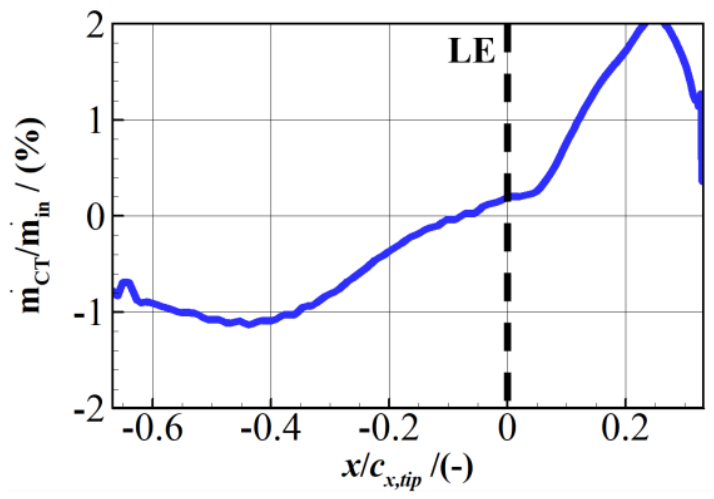

(a) Mass flow distribution

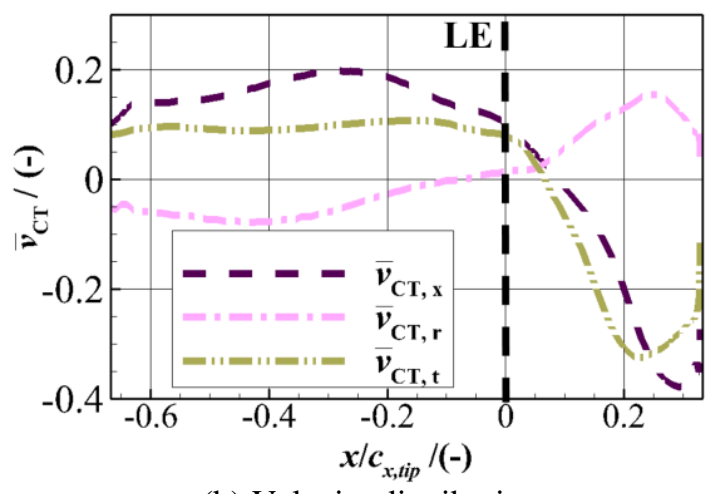

(b) Velocity distribution

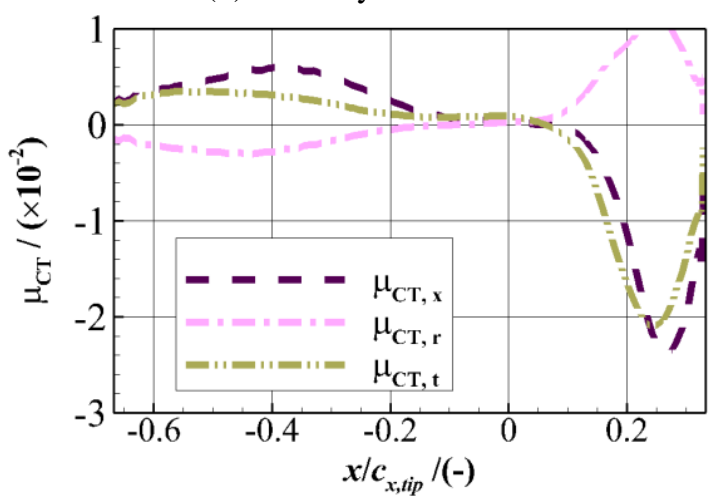

(c) Momentum distribution

\section{Figure 7 Distributions of flow parameters at slots opening}

According to the profile of three parameters, a conceptual schematic of the flow at casing treatment opening is proposed in Figure 8 to manifest it intuitively. The position of leading edge is marked as the demarcation point. Flow is sucked into the slots downstream the leading edge in an obliquely upward direction, while upstream the demarcation, flow injects into the tip region in an obliquely downward direction. Combined with the schematic of tangential velocity in Figure 8 (b), a three-dimensional velocity diagram at the slot opening is obtained in Figure 8 (c).

The profile of mass flow rate, velocity, and momentum on the slots opening has been illustrated to give a new scene of the casing treatment flow. The schematic diagram of velocity is also provided, which can give an intuitive picture of momentum transportation between casing treatment and rotor tip. Based on these, the mechanism of extending stall margin has been drawn.

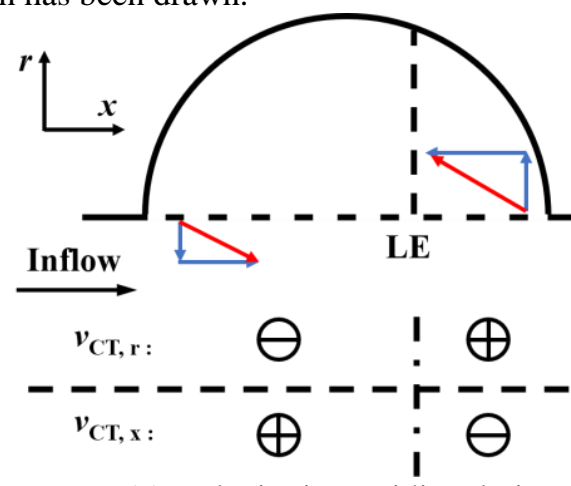

(a) Velocity in meridional view 


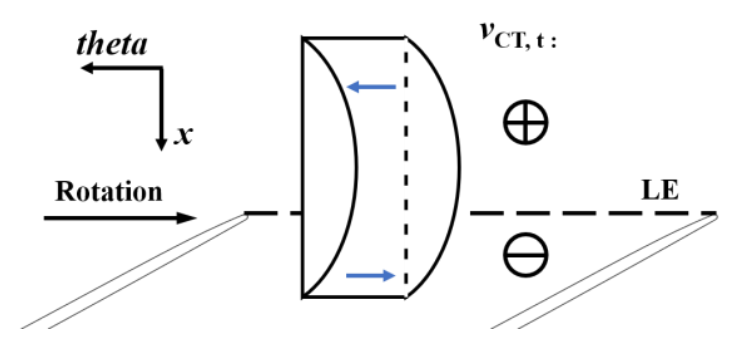

(b) Tangential velocity in pitchwise view

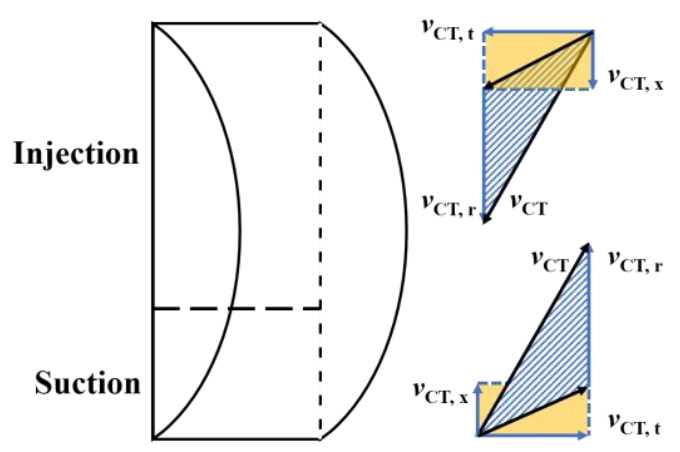

(c) Velocity in 3-D view

\section{Figure 8 Schematic diagram of decomposed velocity}

\subsection{Momentum analysis on CT at different positions}

In this section, the influence of casing treatment with different axial position is inspected. Using the modus introduced in the last section, three types of casing treatment configuration are inspected. The details of configurations have been provided in Section 2 .

The axial momentum distribution can be used as a criterion to evaluate the distance from the stall point. The axial momentum is defined as

$$
\mu_{t i p, x}=\int_{R_{0}}^{R_{1}} \frac{\left|\rho_{t i p} v_{t i p, r}\right| v_{t i p, i}}{\dot{m}_{i n} v_{x, i n} / c_{x, t i p}} d R
$$

where the subscript tip means the tip region. $R$ is the radial distance, and $R_{0}$ is the start of the integration while $R_{I}$ is the end (the detail of integration location is shown in Figure 6).

To obtain the total momentum in slot opening and tip clearance, the total momentum coefficient is defined as

$$
\begin{gathered}
C_{\mu, t i p}=\int_{0}^{1} \frac{\mu_{t i p, i}}{c_{x, t i p}} d x \\
C_{\mu, C T}=\int_{0}^{\frac{x_{C T, \text { end }}}{C_{x, t i p}}} \frac{\mu_{C T, x}}{c_{x, t i p}} d x
\end{gathered}
$$

where variable $x$ is the axial coordinate, and the zero point is at leading edge of the rotor tip, $x_{C T \text {,end }}$ is the axial coordinate of the slot openings end. The details of integration area are shown in Figure 6.

The axial momentum distributions of three configurations have been shown in Figure 9. Compared with the smooth casing configuration, the valley of momentum profile moves downstream in all the three configurations, which confirms the conclusion in reference (Nan, 2014). The tendency of three configurations is similar, with a similar valley value. The valley positions of the distribution are different, with $35 \% c_{x}$, tip in $\mathrm{SE}, 50 \% c_{x}$, tip in $\mathrm{SE}_{\mathrm{M}}$ and $65 \% c_{x}$, tip in $\mathrm{SE}_{\mathrm{L}}$.

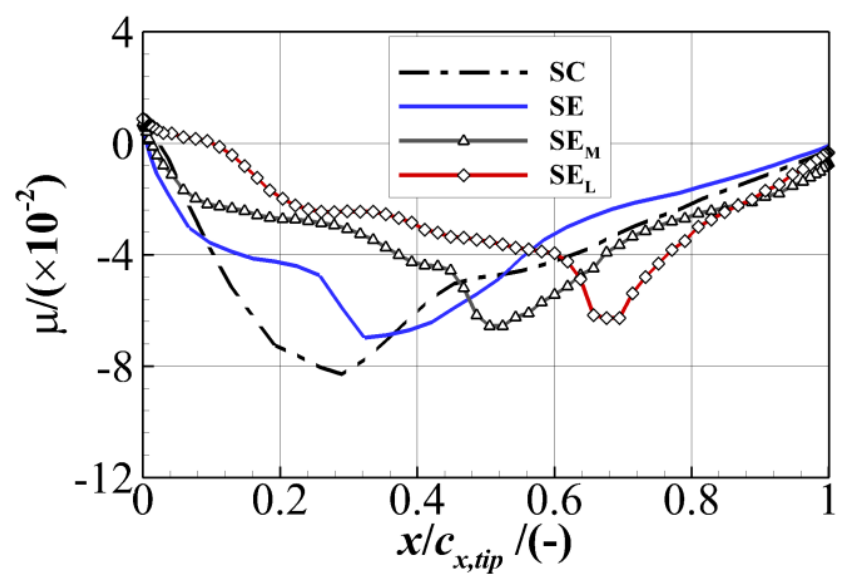

Figure 9 Axial Momentum distributions on tip clearance

The total momentum can be calculated by Equation(6) and(7). Notice that the momentum here is not only on the slot openings, but also on the tip clearance plane. In Figure 10, the variation of axial momentum is displaced, where $\Delta C_{\mu}$ is the variation in tip clearance range and $\Delta C_{\mu, C T}$ is the variation on slots opening. The variation larger than zero means that the change of momentum points toward downstream. As the increase of overlap between casing treatment and blade tip, both $\Delta C_{\mu}$ and $\Delta C_{\mu, C T}$ increase. The momentum of reverse flow descends more with higher overlap between casing treatments and blade tip. However, the experimental result (Danner et al., 2009) show that the configuration SE has the best effect on extending stall margin, which indicates the axial momentum is not the only parameter determines the stall margin.

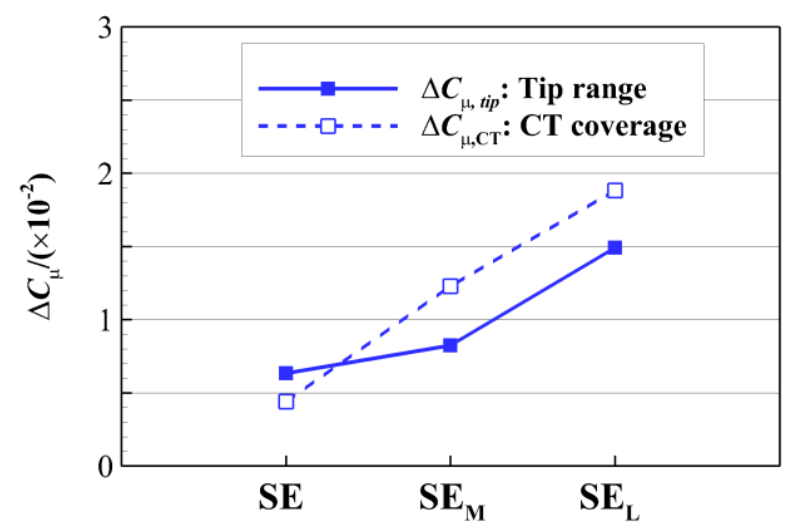

Figure 10 Variation of axial momentum

The momentum on the opening of $\mathrm{SE}_{\mathrm{M}}$ and $\mathrm{SE}_{\mathrm{L}}$ is also decomposed into axial, tangential and radial direction, and the result is shown in Figure 11. The tendency of decomposed 
momentum is similar among three configurations. Three components of velocity reach zero at the same axial position for each configuration, because the mass flow rate there is zero. The position of zero momentum moves downstream as the overlap between rotor and slots increases.

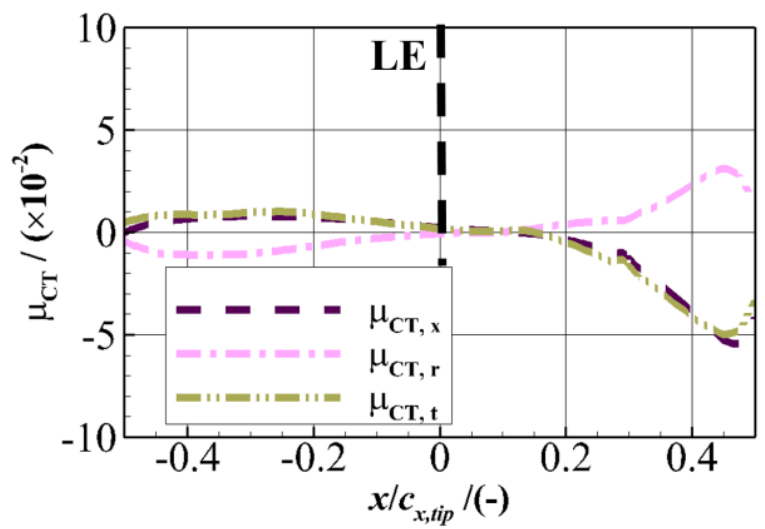

(a) $\mathrm{SE}_{\mathrm{M}}$

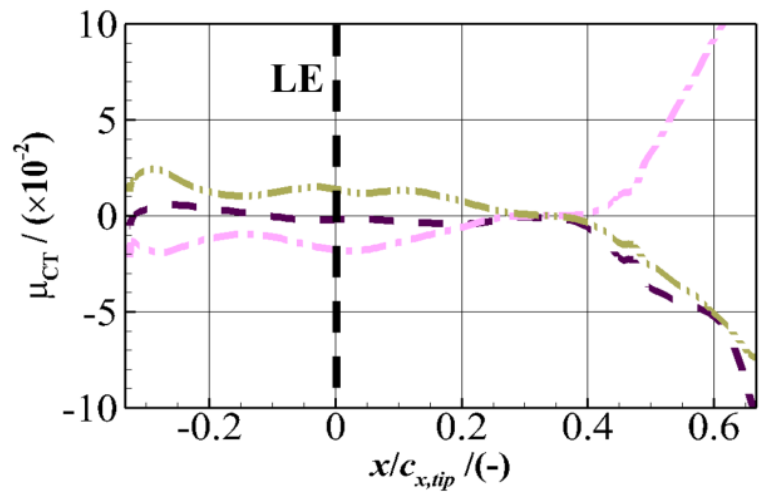

(b) $\mathrm{SE}_{\mathrm{L}}$

Figure 11 Axial momentum distribution on $\mathrm{SE}_{\mathrm{M}}$ and $\mathrm{SE}_{\mathrm{L}}$

The value is completely different on both sides of the zero point. Therefore, this point can be regarded as a demarcation point. The demarcation point has the similar relative position in configuration $\mathrm{SE}_{\mathrm{M}}$ and $\mathrm{SE}_{\mathrm{L}}$, which is at around $40 \% c_{x}$, tip upstream from the end wall of slots. The distribution upstream demarcation point is flat while it is sharp downstream the demarcation point. This is because the suction part is smaller than the injection part. The only way to balance the momentum exchange is to enhance the strength of suction. Though three configurations have similar tendency, the largest value is different. Taking the axial momentum profile for example, the largest value at the suction part of configuration $\mathrm{SE}$ is about $2 \times 10^{-2}$, which is two-fifth of $\mathrm{SE}_{\mathrm{M}}$ and near one fifth of $\mathrm{SE}_{\mathrm{L}}$. This phenomenon is a confirmation of Figure 9. The interaction between casing treatment and tip region is more violent in $\mathrm{SE}_{\mathrm{L}}$ and $\mathrm{SE}_{\mathrm{M}}$ than the original $\mathrm{SE}$. Therefore, the valley of profile in Figure 9 moves downstream. However, as shown by Danner's experimental results, SE has the best stall margin extension among these three configurations. In other words, the stronger suction fails to achieve larger operating range.
The meridional averaged $v_{\mathrm{r}}$ normalized by tip velocity and the velocity vector is illustrated in the Figure 12 . The flow feature is coincident with flow profile above well. For all the three figures, there is a demarcation point at the opening of casing treatment. The suction part is smaller than the injection part, along with higher strength of suction. More than this, Figure 12 is a confirmation of the hypothesis in schematic diagram Figure 8. Upstream the demarcation point, the flow moves into the blade passage and downstream. On the contrary, the flow feature is in stark contrast downstream the

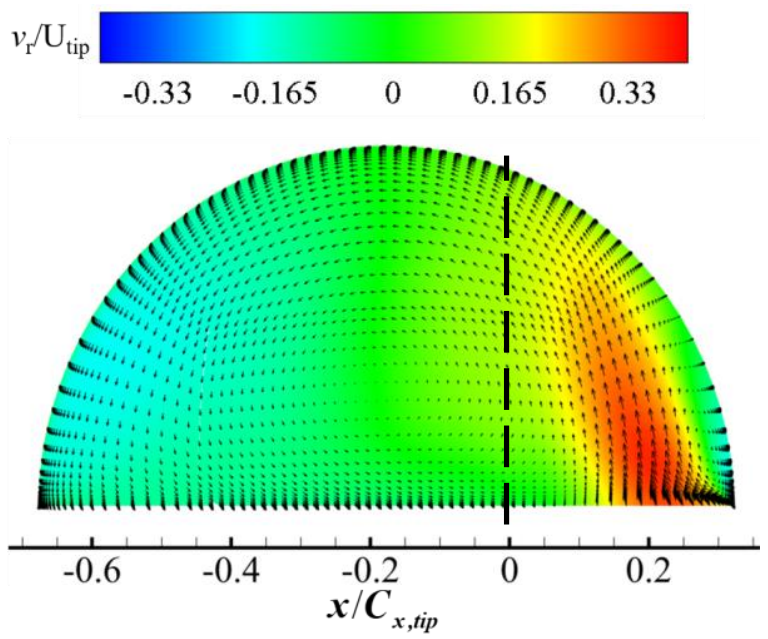

(a) $\mathrm{SE}$

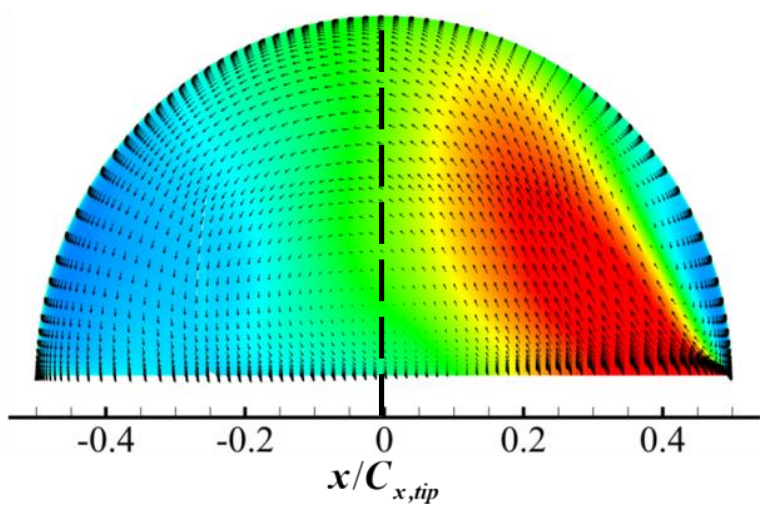

(b) $\mathrm{SE}_{\mathrm{M}}$

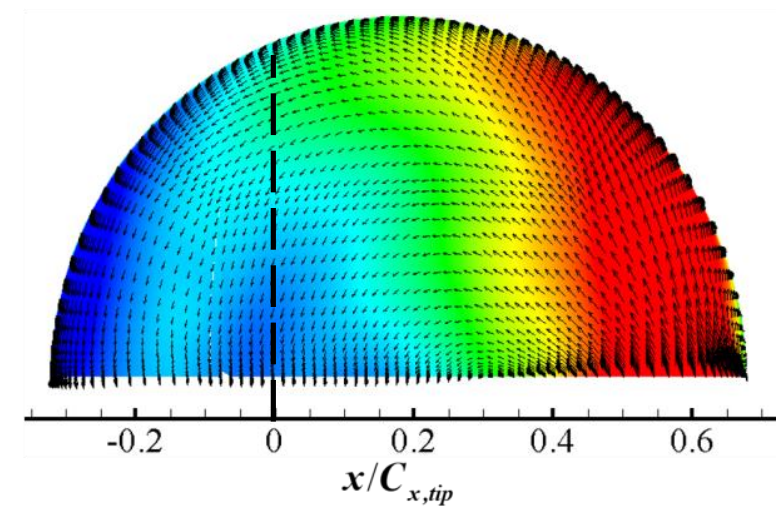

(c) $\mathrm{SE}_{\mathrm{L}}$

Figure 12 Mass flow averaged $v_{r}$ and vector of CT 
demarcation point. It is clear that reverse flow in the tip region is pumped into the casing treatment, which weakens the blockage, and the flow with the positive axial momentum is injected to the tip region to balance the reverse momentum of tip leakage flow. The flow direction in the injection part could be the key point in extending stall margin. In $\mathrm{SE}_{\mathrm{L}}$, the injection is in great strength, but the high component of velocity along the leaning direction of casing treatment could lead to a worse blockage in the tip region. The main purpose is to enhance the axial momentum rather than the radial. In configuration SE, the flow is not violent in radial direction and has a relatively high component in axial direction, which may lead to a better tip flow field.

Inside the casing treatment, the flow is complex. But the overall picture of the flow inside casing treatment is to turn the flow direction. In the configuration $\mathrm{SE}$ and $\mathrm{SE}_{\mathrm{M}}$, a vortex structure is observed at the middle of the slot. Such structure doesn't exist in configuration $\mathrm{SE}_{\mathrm{L}}$.

To compare the axial momentum distribution conveniently, the profiles of three configurations are gathered in Figure 13. The demarcation point on each profile is also marked in the figure. The configuration SE has the largest range where $\mu_{C T, x}$ is larger than 0 upstream the demarcation. For configuration $\mathrm{SE}_{\mathrm{M}}$, the maximum of $\mu_{C T, x}$ is larger than SE. Downstream the demarcation point, $\mathrm{SE}_{\mathrm{L}}$ has the maximum value which can reach over $6 \%$ with reverse direction. In other words, the $\mathrm{SE}_{\mathrm{L}}$ has the best suctioninjection among three configurations. For $\mathrm{SE}_{\mathrm{L}}$, there is a region where $\mu_{C T, x}$ is less than zero upstream the demarcation. Therefore, the injection flow is with negative momentum in this region, which doesn't contribute to extending the stall margin.

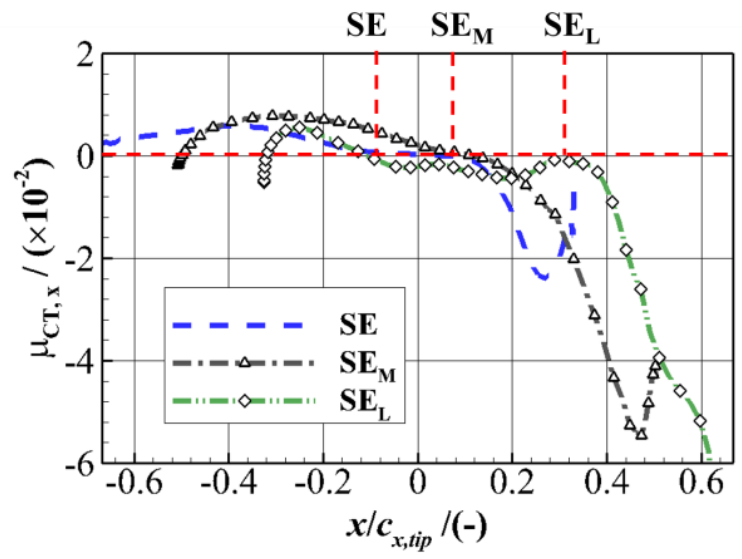

\section{Figure 13 The axial momentum distributions of three configurations}

After the analysis of casing treatment in different axial position, it is found that the total axial momentum is not the only parameter that determines the stall margin extension. Moreover, the suction and injection strength are also not the only parameter for the stall margin extension. So, what's the key point? The distribution of the axial momentum may play an important role here. If the end of injection part is just at the leading edge, the stall margin extension may be the best.
Because the spike inception is caused by the spillage flow at the leading edge and backflow at trailing edge, the axial momentum injected from the casing treatments has the best efficiency in extending the stall margin upstream the leading edge. For the axial momentum downstream the leading edge, it is also effective but it is not as efficient as the upstream one. Besides, as the casing treatment moves downstream, the pressure difference becomes larger, which drives a stronger suction and injection. However, the stronger suction and injection means a stronger radial momentum. As a result, the casing treatment cannot give a higher positive axial momentum at the tip region. Therefore, compared with the $\mathrm{SE}_{\mathrm{L}}, \mathrm{SE}$, and $\mathrm{SE}_{\mathrm{M}}$ have a better margin extension. The best overlap of the casing treatment should be in the range of $33 \%$ to $50 \%$.

In summary, the momentum distribution shows the general character in three configurations. There is still a difference in each configuration. The distribution profile of SE is flat, and the profiles of $\mathrm{SE}_{\mathrm{M}}$ and $\mathrm{SE}_{\mathrm{L}}$ are relatively sharp. Through the analysis, the ability of suction and injection is evaluated in quantity. A hypothesis in designing casing treatment has been proposed. In other words, the best location of casing treatments is to make the region of injection part just upstream the leading edge of rotor blade.

\section{CONCLUSIONS}

In this paper, the steady and unsteady numerical simulation is conducted for a transonic rotor with and without axial-slot casing treatment. Momentum transportation analysis is performed at both conditions. Then, the influence of casing treatment axial position is also investigated. By momentum transportation analysis, the interaction between casing treatment and tip region has been illustrated. Finally, a hypothesis for design is proposed. The analysis conclusions are shown as follow:

1) The momentum distribution on tip clearance can reveal the trajectory of tip leakage flow clearly. The changes in tip leakage flow with and without casing treatments can give an explanation for the stall margin extension. With casing treatment, the flow with positive momentum is injected upstream the leading edge and weakens the blockage caused by tip leakage flow. The maximum value of inverse momentum decreases from 0.5 to less than 0.45 and the maximum value of positive momentum increase to 0.198 .

2) The momentum transportation analysis on slots opening indicates the flow interaction between casing treatments and tip region. A schematic diagram of the flow velocity is obtained. There is a demarcation point at the slot opening, dividing the slot opening into the injection and the suction part. In the injection part, the flow is of positive momentum and injected into the tip region. In the suction part, the flow is of reverse momentum and pumped into the casing treatments.

3) The axial position of casing treatment has an effect on the suction-injection ability. As the casing treatment moves downstream, the suction-injection ability becomes stronger. For the most downstream configuration $\mathrm{SE}_{\mathrm{L}}$, the maximum axial momentum can reach about 0.02 , which is 5 times larger 
than the momentum of SE. However, the effect on improving the stall margin doesn't have a linear relation with suctioninjection ability. The configuration which makes the region of injection part just upstream the leading edge may have the best effect on stall margin extension. This hypothesis needs to be validated in future work.

\section{NOMENCLATURE}

$\alpha$

$\pi$

$\rho$

$v$

$\dot{m}_{\text {in }}$

$c_{x, \text { tip }}$

$\tau$

$C_{P}$

$P$

$P_{\text {in }}$

$P^{*}$

$L$

$\bar{v}_{C T, i}$

$\mu_{C T, i}$

$\mu_{t i p, x}$

$C_{\mu, t i p}$

$C_{\mu, C T}$

$\Delta C_{\mu, t i p}$

$\Delta C_{\mu, C T}$
Normalized momentum distribution per unit area

Absolute total pressure ratio

Density of flow

velocity of flow

Mass flow rate at inlet plane

Axial chord length of tip region

Pitch distance between two rotor tips

Static pressure coefficient

Static pressure

Static pressure at inlet plane

Total pressure

circumferential width of slot

Normalized $i$ direction velocity on slots opening where $i$ can be $x$ (axial), $r$ (radial), $t$ (tangential)

Normalized $i$ direction momentum distribution per unit length on slots opening where $i$ can be $x$ (axial), $r$ (radial), $t$ (tangential)

Normalized axial momentum distribution per unit length at rotor tip

Total axial momentum at rotor tip

Total axial momentum on slot openings

Variation of total axial momentum at tip

Variation of total axial momentum on slot openings

$\begin{array}{ll}\text { Abbreviation } & \\ \text { CT } & \text { Casing treatment } \\ \text { SC } & \text { Smooth casing } \\ \text { SE } & \text { Semi-circular casing treatment } \\ \text { SE }_{M} & \text { Middle semi-circular casing treatment } \\ \mathrm{SE}_{\mathrm{L}} & \text { Large semi-circular casing treatment } \\ \mathrm{S.M} . & \text { Stall margin } \\ \mathrm{SIM} & \text { Simulation } \\ \mathrm{EXP} & \text { Experiment } \\ \text { uns } & \text { Unsteady } \\ x(\text { variable }) & \text { Axial distance from leading edge }\end{array}$

$\begin{array}{ll}\text { Subscript } & \\ s & \text { Stall condition } \\ d & \text { Design condition } \\ \text { in } & \text { Inlet of computational domain } \\ t i p & \text { Tip region } \\ x & \text { Axial direction } \\ r & \text { Radial direction } \\ t & \text { Tangential direction }\end{array}$

\section{ACKNOWLEDGMENTS}

The authors gratefully acknowledge the supports of Natural Science Foundation of China (No. 51576124), National Science and Technology Major Project (2017-II0004-0017), and Initiative Postdocs Supporting Program (No.18Z102060064). Particular thanks go to Mr Maximilian Jüngst and the Institute of Gas Turbines and Aerospace Propulsion in Technische Universität Darmstadt, who offered the geometry of the compressor stage and presented detailed experiment results in previous researches.

\section{References}

[1] Bailey, E. E. and Voit, C. H. (1970). Some observations of effects of porous casings on operating range of a single axial-flow compressor rotor. NASA TM X-2120.

[2] Brandstetter, C., Wartzek, F., Werner, J., Schiffer, H.-P. and Heinichen, F. (2015). Unsteady measurements of periodic effects in a transonic compressor with casing treatments. ASME Paper No. GT2015-42394.

[3] Cameron, J. D., Bennington, M. A., Ross, M. H., Morris, S. C., Du, J., Lin, F. and Chen, J. (2013). The influence of tip clearance momentum flux on stall inception in a high-speed axial compressor. Journal of Turbomachinery, 135(5), 051005 .

[4] Danner, F. C., Kau, H.-P., Muller, M. M., Schiffer, H.P. and Brignole, G. A. (2009). Experimental and numerical analysis of axial skewed slot casing treatments for a transonic compressor stage. ASME Paper No. GT2009-59647.

[5] Du, J., Lin, F., Zhang, H. and Chen, J. (2010). Numerical investigation on the self-induced unsteadiness in tip leakage flow for a transonic fan rotor. Journal of Turbomachinery, 132(2), 021017.

[6] Emmrich, R., Kunte, R., Honen, H. and Niehuis, R. (2007). Time Resolved Investigations of an Axial Compressor With Casing Treatment: Part 2-Simulation. ASME Paper No. GT2007-27582.

[7] Hewkin-Smith, M., Pullan, G., Grimshaw, S., Greitzer, E. and Spakovszky, Z. (2019). The role of tip leakage flow in spike-type rotating stall inception. Journal of Turbomachinery, 141(6), 061010.

[8] Koch, C. (1970). Experimental evaluation of outer case blowing or bleeding of single stage axial flow compressor, part 6 Final report. NASA CR-54592.

[9] Lu, X., Chu, W., Zhu, J. and Zhang, Y. (2009). Numerical investigations of the coupled flow through a subsonic compressor rotor and axial skewed slot. Journal of Turbomachinery, 131(1), 011001.

[10] Moore, R. D., Kovich, G. and Blade, R. J. (1971). Effect of casing treatment on overall and blade element performance of a compressor rotor. NASA TN D-6538.

[11] Muller, M. W., Biela, C., Schiffer, H.-P. and Hah, C. (2008). Interaction of rotor and casing treatment flow in an axial single-stage transonic compressor with circumferential grooves. ASME Paper No. GT2008-50135.

[12] Nan, X. 2014. The compressor rotor tip control volume method and its application on circumferential groove casing treatment. PhD thesis, University of Chinese Academy of Sciences.

[13] Ross, M. H., Cameron, J. D., Morris, S. C., Chen, H. and Shi, K. (2017). Axial Compressor Stall, Circumferential 
Groove Casing Treatment, and the Tip-Clearance Momentum Flux. Journal of Propulsion and Power, 34(1), 146-152.

[14] Shabbir, A. and Adamczyk, J. J. (2005). Flow mechanism for stall margin improvement due to circumferential casing grooves on axial compressors. Journal of turbomachinery, 127(4), 708-717.

[15] Vo, H. D., Tan, C. S. and Greitzer, E. M. (2008). Criteria for spike initiated rotating stall. Journal of turbomachinery, 130(1), 011023.

[16] Wilke, I. (2004). A numerical investigation of the flow mechanisms in a high pressure compressor front stage with axial slots. Journal of Turbomachinery, 126(3), 339-349. 